UR-1531

$\mathrm{ER} / 40685 / 918$

VPI-IPPAP-98-2

May 1998

\title{
Two-Higgs-Doublet-Models and Radiative CP Violation ${ }^{\star}$
}

\author{
Otto C.W. Kong* and Feng-Li Lin ${ }^{\dagger}$ \\ * Department of Physics and Astronomy, \\ University of Rochester, Rochester NY 14627-0171. \\ $\dagger$ Department of Physics, and Institute for Particle Physics and Astrophysics, \\ Virginia Polytechnic Institute and State University, Blacksburg, VA 24061-0435.
}

\begin{abstract}
We discuss the feasibility of spontaneous $\mathrm{CP}$ violation being induced by radiative corrections in 2HDM's. Specifically, we analyze the cases of gaugino/higgsino effect on MSSM, and a new model with an extra exotic quark doublet. The new model, while demonstrating well the Georgi-Pais theorem, is also expected to be phenomenlogically interesting.
\end{abstract}

* Talk given by O.K. at MRST conference, McGill University, Montréal, May 1998

- submission for proceedings. 


\title{
Two-Higgs-Doublet-Models and Radiative CP Violation ${ }^{1}$
}

\author{
Otto C.W. Kong* and Feng-Li Lin ${ }^{\dagger}$ \\ * Department of Physics and Astronomy, \\ University of Rochester, Rochester NY 14627-0171. \\ $\dagger$ Department of Physics, and Institute for Particle Physics and Astrophysics, \\ Virginia Polytechnic Institute and State University, Blacksburg, VA 24061-0435.
}

\begin{abstract}
We discuss the feasibility of spontaneous $\mathrm{CP}$ violation being induced by radiative corrections in 2HDM's. Specifically, we analyze the cases of gaugino/higgsino effect on MSSM, and a new model with an extra exotic quark doublet. The new model, while demonstrating well the Georgi-Pais theorem, is also expected to be phenomenlogically interesting.
\end{abstract}

\section{INTRODUCTION}

The source of $\mathrm{CP}$ violation is one of the most important unsolved puzzles in particle physics. On the one hand, CP violation is observed experimentally only in the $K^{0}-\bar{K}^{0}$ system, with the corresponding weak $\mathrm{CP}$ phase compatible with the Kobayashi-Maskawa (KM) mechanism. On the other hand, the experimental bound on the neutron electric dipole moment indicates that the effective strong $\mathrm{CP}$ phase has to be exceedingly small,

$$
\bar{\theta}<10^{-9} .
$$

The origin of this strong CP problem lies in the necessity of adding the so-called $\theta$ term to the effective QCD Lagrangian due to the contribution of instantons present in the topologically nontrivial $\mathrm{QCD}$ vacuum :

$$
\mathcal{L}_{\text {eff }}=\frac{\theta \alpha_{s}}{8 \pi} F_{\mu \nu}^{A} \tilde{F}^{A \mu \nu},
$$

where the dual field strength is given by $\tilde{F}_{\mu \nu}=\frac{1}{2} \epsilon_{\mu \nu \alpha \beta} F^{\alpha \beta}$. Through the anomaly in the QCD axial U(1) current, chiral U(1) transformations lead to shifts in $\theta$, leaving

1) Talk presented by O.K.. 
the physical combination $\bar{\theta}=\theta-\arg \operatorname{det} M_{q}$, where $M_{q}$ is the quark mass matrix, as the effective strong $\mathrm{CP}$ phase. In the supersymmetrized version of the standard model, new CP phases from supersymmetry (SUSY) breaking terms also have to be small $\left(<10^{-3}\right)$. Spontaneously CP violation (SCPV) provides an elegant theory with a good control on the magnitude of the various CP phases. Hence models of SCPV keep generating new interest.

\section{SCPV IN A TWO-HIGGS-DOUBLET MODEL}

The most simple setting for achieving the SCPV scenario is given by a twoHiggs-doublet model (2HDM) [2]. The most general scalar potential for two Higgs doublets, $\phi_{1}$ and $\phi_{2}$, is given by

$$
\begin{aligned}
V\left(\phi_{1}, \phi_{2}\right)= & m_{1}^{2} \phi_{1}^{\dagger} \phi_{1}+m_{2}^{2} \phi_{2}^{\dagger} \phi_{2}-\left(m_{3}^{2} \phi_{1}^{\dagger} \phi_{2}+\text { h.c. }\right) \\
& +\lambda_{1}\left(\phi_{1}^{\dagger} \phi_{1}\right)^{2}+\lambda_{2}\left(\phi_{2}^{\dagger} \phi_{2}\right)^{2}+\lambda_{3}\left(\phi_{1}^{\dagger} \phi_{1}\right)\left(\phi_{2}^{\dagger} \phi_{2}\right) \\
& +\lambda_{4}\left(\phi_{1}^{\dagger} \phi_{2}\right)\left(\phi_{2}^{\dagger} \phi_{1}\right)+\frac{1}{2}\left[\lambda_{5}\left(\phi_{1}^{\dagger} \phi_{2}\right)^{2}+\text { h.c. }\right] \\
& +\frac{1}{2}\left\{\phi_{1}^{\dagger} \phi_{2}\left[\lambda_{6}\left(\phi_{1}^{\dagger} \phi_{1}\right)+\lambda_{7}\left(\phi_{2}^{\dagger} \phi_{2}\right)\right]+\text { h.c. }\right\} .
\end{aligned}
$$

Assuming all the parameters in $V$ being real, and denoting the vacuum expectation values (VEV's) of the neutral components of the Higgs doublets by

$$
\left\langle\phi_{1}^{0}\right\rangle=v_{1} \quad \text { and } \quad\left\langle\phi_{2}^{0}\right\rangle=v_{2} e^{i \delta}
$$

we have

$$
\begin{aligned}
\langle V\rangle= & m_{1}^{2} v_{1}^{2}+m_{2}^{2} v_{2}^{2}+\lambda_{1} v_{1}^{4}+\lambda_{2} v_{2}^{4}+\left(\lambda_{3}+\lambda_{4}-\lambda_{5}\right) v_{1}^{2} v_{2}^{2} \\
& +2 \lambda_{5} v_{1}^{2} v_{2}^{2} \cos ^{2} \delta-\left(2 m_{3}^{2}-\lambda_{6} v_{1}^{2}-\lambda_{7} v_{2}^{2}\right) v_{1} v_{2} \cos \delta \\
= & M_{1} v_{1}^{2}+M_{2} v_{2}^{2}+\left(p v_{1}^{4}+2 r v_{1}^{2} v_{2}^{2}+q v_{2}^{4}\right) \\
& +2 \lambda_{5} v_{1}^{2} v_{2}^{2}(\cos \delta-\Omega)^{2}-\frac{m_{3}^{4}}{2 \lambda_{5}}
\end{aligned}
$$

where

$$
\Omega=\frac{2 m_{3}^{2}-\lambda_{6} v_{1}^{2}-\lambda_{7} v_{2}^{2}}{4 \lambda_{5} v_{1} v_{2}}
$$

and

$$
\begin{aligned}
& M_{1}=m_{1}^{2}+\frac{\lambda_{6} m_{3}^{2}}{2 \lambda_{5}}, \\
& M_{2}=m_{2}^{2}+\frac{\lambda_{7} m_{3}^{2}}{2 \lambda_{5}}
\end{aligned}
$$




$$
\begin{aligned}
p & =\lambda_{1}-\frac{\lambda_{6}^{2}}{8 \lambda_{5}}, \\
q & =\lambda_{2}-\frac{\lambda_{7}^{2}}{8 \lambda_{5}}, \\
r & =\frac{1}{2}\left(\lambda_{3}+\lambda_{4}-\lambda_{5}-\frac{\lambda_{6} \lambda_{7}}{4 \lambda_{5}}\right) .
\end{aligned}
$$

A nontrivial phase $(\delta)$ then indicates SCPV.

Let us look at the $\delta$-dependence of $\langle V\rangle$. The extremal condition gives

$$
-4 \lambda_{5} v_{1}^{2} v_{2}^{2}(\cos \delta-\Omega) \sin \delta=0,
$$

and the stability condition requires

$$
\frac{\partial^{2} V}{\partial \delta^{2}}=4 \lambda_{5} v_{1}^{2} v_{2}^{2}\left[\cos \delta(\Omega-\cos \delta)+\sin ^{2} \delta\right]>0 .
$$

$\cos \delta=\Omega$ gives a SCPV solution, provided that $\lambda_{5}>0$ and $|\Omega|<1$. Actually, Eq.(2) shows that this is the absolute minimum. In order for $\mathrm{V}$ to have a lower bound, we have the extra constraints

$$
p>0, \quad q>0,
$$

and

$$
p q>\left[r+\lambda_{5}(\cos \delta-\Omega)^{2}\right]^{2} .
$$

The latter reduced to

$$
p q>r^{2}
$$

for the $\mathrm{CP}$ violating minimum.

However, in order to avoid flavor-changing-neutral-currents that could result, extra structure like natural flavor conservation (NFC) [3] has to be imposed on a 2HDM, which then forbids SCPV [4]. For instance, a natural way to impose NFC is to require that only one of the Higgs, say $\phi_{1}$, transforms nontrivially under an extra discrete symmetry. This means that $m_{3}^{2}, \lambda_{6}$ and $\lambda_{7}$, and may be $\lambda_{5}$ too, all have to vanish. Similarly, a supersymmetric version of the standard model (SM) is naturally a $2 \mathrm{HDM}$ with NFC being imposed automatically by the holomorphy of the superpotential. The tree level scalar potential there has vanishing $\lambda_{5}, \lambda_{6}$, and $\lambda_{7}$, though the soft SUSY breaking $B$-term gives rise to a nonvanishing $m_{3}^{2}$. The interesting point of concern then is whether radiative corrections can modify the picture. Note that a positive $\Delta \lambda_{5}$ is needed for this radiative CP violation scenario. 


\section{RADIATIVE CP VIOLATION}

For the case of the minimal supersymmetric standard model (MSSM), Maekawa

[5] pointed out that there is a positive contributions to $\lambda_{5}$ from a finite 1-loop diagram (Fig. 1a) involving the gauginos and higgsinos, which could lead to SCPV. However, there are objections to the particular scenario. Maekawa [5] realized that the situation will not be able to give rise to sufficient weak CP vioation for the $K^{0}$ $\bar{K}^{0}$ system. Pomarol [6] argued that mass for the "psuedoscalar", $m_{A}$, is roughly proportional to $\sqrt{\lambda_{5}}$ and is at least more than a factor of three too small to be phenomenologically acceptable. Actually, we worked out the algebra to decoupled the Goldstone mode explicitly assuming the CP violating vacuum, and got the following results [1]: the $3 \times 3$ physical Higgs mass-squared matrix $m_{H i j}^{2}$ gives

$$
\operatorname{det}\left(m_{H}^{2}\right)=\lambda_{5}\left(p q-r^{2}\right) \sin ^{2} 2 \beta \sin ^{2} \delta ;
$$

as $p=q=-r>0$

at tree level, when only a positive $\Delta \lambda_{5}$ is considered, $\left(p q-r^{2}\right)$ and hence $\operatorname{det}\left(m_{H}^{2}\right)$ becomes negative [see Eq.(8)]. In fact, with a negative $r,\left(p q<r^{2}\right)$ exactly violates the condition for the potential to be bounded from below. This inconsistency is also pointed out by Haba [7], who then suggested that it would be fixed when top/stop loop contributions to the other parameters, mainly $\lambda_{2}$, in the potential are included. From our perspective, in order to have a consistent approximation to the radiative corrections in whatever interesting region of the parameter space, loop contributions to all parameters in the scalar potential at the same order have to be considered. Corrections to all
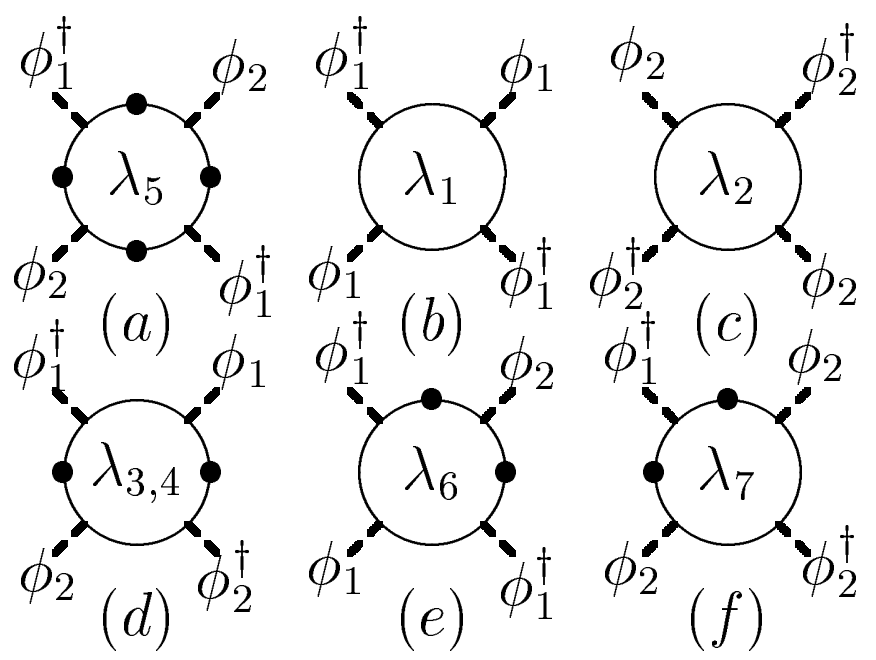

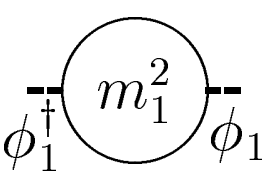

$(g)$

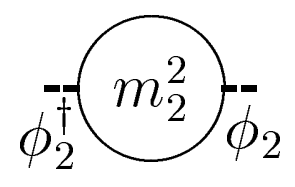

(h)

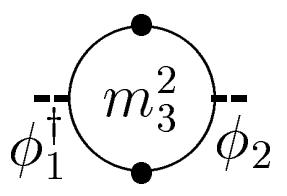

(i)

FIGURE 1. Gaugino/higgsino-loop diagrams giving rise to modifications to parameters in the potential. (Each dot indicates a helicity flip in the fermion propagator.) parameters in $V$ do exists, as shown in Fig. 1. 


\section{A NEW MODEL WITH AN EXOTIC QUARK DOUBLET}

Before going into discussion of a consistent 1-loop treatment, we first present a new model introduced in Ref. [1] that we believe could be experimentally viable. Our new model has an extra pair of vectorlike quark doublets, $Q$ and $\bar{Q}$, with the following couplings

$$
\mathcal{L}_{Q}=M_{Q} \bar{Q} Q+\lambda_{Q} \bar{t} \phi_{1}^{\dagger} Q
$$

as an addition to the two-Higgs-doublet SM or MSSM. Note that $\phi_{1}^{\dagger}$ is actually $H_{d}$, the Higgs (super)multiplet that gives masses to the down-type quarks; and $\phi_{2}$ is $H_{u}$. So, $T_{3}=-1 / 2$ component of $Q$, denoted by $T$, has the same charge as the top quark and mixes with it after electroweak (EW) symmetry breaking. The other part of the doublet is a quark of electric 5/3. The 1-loop diagram, now with the gaugino/higgsino propagators replaced by that of the quarks, leads to $\Delta \lambda_{5}\left(\sim 3 \lambda_{Q}^{2} \lambda_{t}^{2} / 16 \pi^{2}\right)$ and could be very substantial for large Yukawa couplings. (Note that $\phi_{1}$ and $\phi_{2}$ vertices now have $\lambda_{Q}$ and $\lambda_{t}$ couplings respectively).

The mass matrix of the $t$ - $T$ system is given by

$$
\mathcal{M}_{t}=\left(\begin{array}{cc}
\lambda_{t}\left\langle\phi_{2}\right\rangle & \lambda_{Q}\left\langle\phi_{1}^{\dagger}\right\rangle \\
0 & M_{Q}
\end{array}\right)
$$

Notice that the model actually has an effective KM phase to account for weak CP phenomenology. Assuming $M_{Q}$ to be roughly around the same order as the EW scale, the model can also easily get around the "small $m_{A}$ " objection. Moreover, its modification to top quark phenomenology would be very interesting, and will provide an experimental check on its viability. The $Q-\bar{Q}$ exotic quarks could naturally arise, for example, as the only extra quarks from some interesting models with a SM-like chiral fermion spectra embedding the three SM families in a intriguing way [8].

\section{A CONSISTENT 1-LOOP TREATMENT AND THE GEORGI-PAIS THEOREM}

Here we give a brief discussion of our consistent 1-loop treatment for both MSSM and the new model, particularly in relation to the Georgi-Pais theorem [9]. Readers are referred to our original paper [1] for more details.

The Georgi-Pais theorem states that radiative $\mathrm{CP}$ violation can occur if and only if there exists spinless bosons which are massless in the tree approximation. Naively looking, the above radiative $\mathrm{CP}$ violation pictures violate the theorem. There are some confusing statement in the literature in relation to the situation. However, the theorem has a presumption, that no fine tuning be allowed. Results from our 


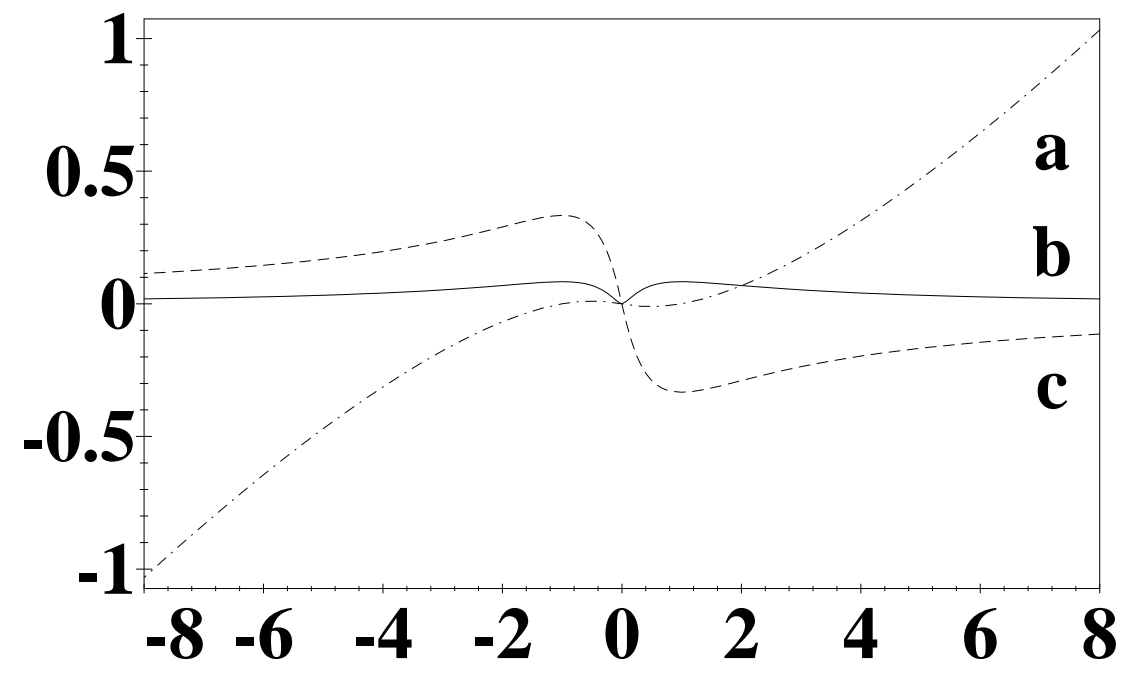

FIGURE 2. Plots of radiative correction from chargino loop verses $m\left(=M_{\tilde{g}} / \mu\right)$, with mass mixing from EW symmetry neglected. (a) $\Delta m_{3}^{2}(\overline{M S})$ in $25 \times g^{2} \mu^{2} / 16 \pi^{2}$; (b) $\Delta \lambda_{5}$; (c) $\Delta \lambda_{6}\left(=\Delta \lambda_{7}\right)$; both of the latter curves with values in $g^{4} / 16 \pi^{2}$.

consistent treatment of the two models discussed to be sketched below show exactly that some form of fine tuning is unavoidable for radiative $\mathrm{CP}$ violation to occur in both cases. There has also been statements about the smallness of $m_{A}$ being a necessary consequence of the Georgi-Pais theorem. Comparison between the two models dicsussed here clearly illustrated that is not true. Smallness of $m_{A}$ in the MSSM cases is rather the result of the small gauge couplings used to produce $\Delta \lambda_{5}$.

A positive $\Delta \lambda_{5}$ is a necessary but not sufficient condition for radiative CP violation. This is clearly illustrated in our discussion of the scalar potential and the problem of the Maekawa picture. In our opinion, it is at least of theoretical interest to see what the 1-loop gaugino/higgsino effect alone could do to the vacuum solution of a supersymmetric 2HDM. A consistent treatment of the 1-loop effect should of course take into consideration contributions to all the 10 parameters in the potential $V$. Recall that another essential condition for the existence of the CP violating vacuum solution is $|\Omega|<1$.

We plot the numerical results of major interest in Fig.2. The plots are for the chargino contributions only, as functions of $m=M_{\tilde{g}} / \mu$, the gaugino-higgsino mass 
ratio. Our results here presented give the 1-loop effect before EW symmetry breaking, i.e. mass mixing between the gaugino and higgsino were not considered. Further modifications due to the symmetry breaking are not expected to change the general features. Here, the neutralino contributions can simply be inferred from symmetry.

Taking the renormalized value of $m_{3}^{2}$ as a free parameter, it has a magnitude that increases fast with that of $m$ for $|m|>1$. Obviously, some fine tuning is needed to get $|\Omega|<1$, though a small window on $m$ always exists not too far from $|m|=1$, for each sign of $\mu$, for a not too small $\mu$. With EW-scale $\mu, \Delta m / m$ of the admissible regions are of order $10^{-2}$, though the severe fine tuning can be tamed by having small $\mu$.

In the our new model, though the new quark doublet $Q$ has mass before EW symmetry breaking, a similar set of 1-loop diagrams, as those given in Fig.1, can only be completed when the EW breaking mass of the top and its mixing with $T$ are taken into consideration. But then the plausibly large Yukawa couplings give

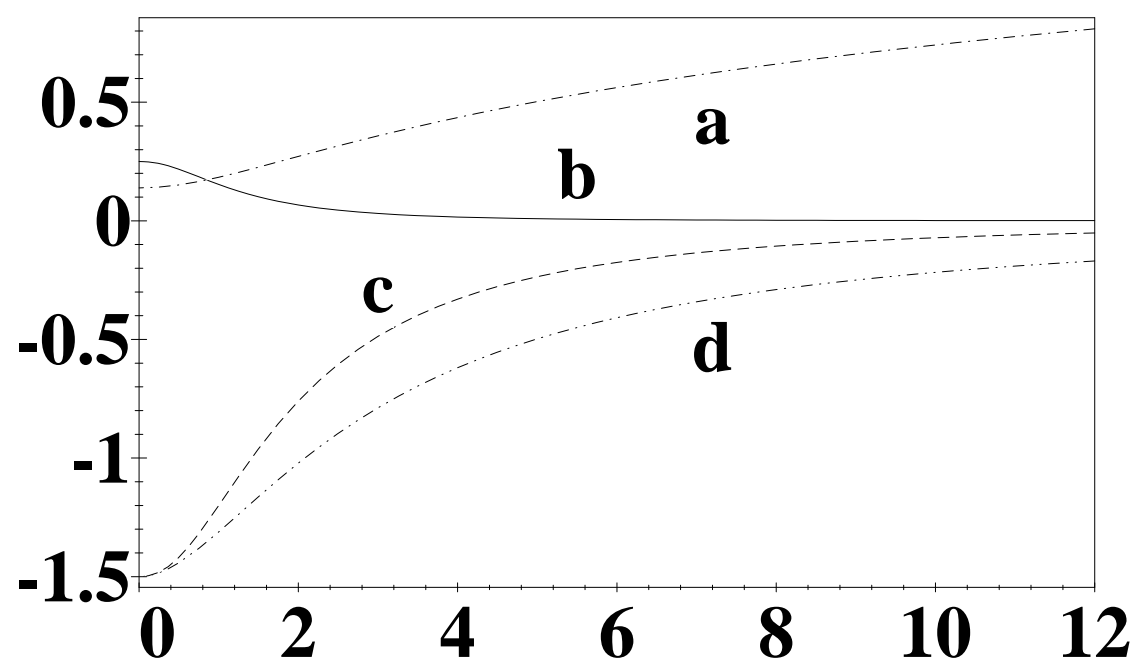

FIGURE 3. Plots of radiative correction from $t$ - $T$ loop verses $m\left(=M_{Q} / v_{2}\right)$. (a) $\Delta m_{3}^{2}(\overline{M S})$ in $10 \times 3 \lambda_{t}^{2} v_{2}^{2} / 16 \pi^{2}$; (b) $\Delta \lambda_{5}$; (c) $\Delta \lambda_{6}$; (d) $\Delta \lambda_{7}$; all of the latter three curves with values in $3 \lambda_{t}^{4} / 16 \pi^{2}$; ( $\lambda_{t}=\lambda_{Q}, \tan \beta=1$ assumed $)$. 
rise to substantial results. In Fig.3, we presented some numerical results. The plots use again $m$ as parameter which in this case denotes $M_{Q} / v_{2}$. For simplicity, we assume $\lambda_{Q}=\lambda_{t}$.

Here , $\Delta m_{3}^{2}(\overline{M S})$ always has the opposite sign to that of $\Delta \lambda_{6}$ or $\Delta \lambda_{7}$, making a naive use of the value to fit in the $|\Omega|<1$ condition impossible. Hence, for SCPV to occur, a tree level $m_{3}^{2}$ value is needed. While the $m_{3(\text { tree })}^{2}$ then has to be chosen to roughly match the $t$ - $T$ 1-loop effect, the large Yukawa couplings make this relatively natural, as a value of the order $v_{2}$ is all required. Accepting that, $\Delta m_{3(\text { tree })}^{2} / m_{3 \text { (tree })}^{2}$ of the solution region is not quite small, $\gtrsim .35$ for $m \leq 1$, representing only a moderate fine tuning. The small $m_{3}^{2}$ required is natural, as its zero limit provides the scalar potantial with an extra Peccei-Quinn type symmetry.

\section{CONCLUSION}

We have performed a consistent 1-loop analysis of the feasibility of radiatively induced SCPV, for both the MSSM and our proposed new model with a pair of vector-like exotic quark doublets. Our results clarified some issues concerned. The new model, especially a SUSY version, is expected to be phenomenologically interesting. Though a complete studies of its various features and experimental viability still have to be performed, we have illustrated at least how it can easily overcome the objections to the corresponding scenario within MSSM. Further studies of the model is under progress.

\section{REFERENCES}

1. Kong, O.C.W., and Lin, F.-L., Phys. Lett. B 419, 217 (1998).

2. Lee, T.D., Phys. Rev. D 8, 1226 (1973).

3. Glashow, S.L., and Weinberg, S., Phys. Rev. D 15, 1958 (1977).

4. Branco, G.C., Phys. Rev. D 22, 2901 (1980).

5. Maekawa, N., Phys. Lett. B 282, 387 (1992).

6. Pomarol, A., Phys. Lett. B 287, 331 (1992).

7. Haba, N., Phys. Lett. B 398, 305 (1997).

8. Kong, O.C.W., Mod. Phys. Lett. A11, 2547 (1996); Phys. Rev. D 55, 383 (1997).

9. Georgi, H., and Pais, A., Phys. Rev. D10, 1246 (1974). 